\title{
Role of TFEB-driven autophagy regulation in pancreatic cancer treatment
}

\author{
KATHRIN KLEIN $^{1 *}$, KRISTIN WERNER $^{1 *}$, CHRISTIAN TESKE ${ }^{1}$, MIRIAM SCHENK $^{2}$, \\ THOMAS GIESE $^{3}$, JÜRGEN WEITZ ${ }^{1}$ and THILO WELSCH ${ }^{1}$
}

\author{
${ }^{1}$ Department of Visceral, Thoracic and Vascular Surgery, University Hospital Carl Gustav Carus, \\ Technische Universität Dresden, D-01307 Dresden; ${ }^{2}$ Department of General, Visceral and Transplantation Surgery, \\ ${ }^{3}$ Institute for Immunology, University of Heidelberg, D-69120 Heidelberg, Germany
}

Received February 22, 2016; Accepted April 4, 2016

DOI: 10.3892/ijo.2016.3505

\begin{abstract}
Autophagy pathways promote the growth of pancreatic ductal adenocarcinoma (PDAC), but the critical role is yet to be determined. Transcription factor EB (TFEB) centrally controls lysosomal and autophagy biogenesis. This study aimed to explore the role of TFEB for autophagy regulation in PDAC. We found that TFEB expression was significantly elevated in human PDAC samples $(n=45)$, and localized to the cytoplasm and nucleus in 11 of 15 cases. In primary PDAC cell lines, TFEB nuclear expression was evident even under basal conditions, and further nuclear enrichment was achieved by starvation. Transient RNA interference reduced TFEB expression to $11-23 \%$, but starvation-induced accumulation of the lipidated, autophagosome-associated LC3-II and the autophago-to-lysosome route was maintained after TFEB silencing. Likewise, gemcitabine treatment of the cancer cells augmented apoptosis and LC3-II as an indicator of autophagy, regardless of the TFEB expression levels. Moreover, the interplay of oncogenic KRAS with TFEB and autophagy was investigated. KRAS silencing caused PDAC cell apoptosis and a reciprocal increase in TFEB expression. This inverse correlation could be confirmed in published data sets of genetically engineered mouse models and human PDAC samples using the the Pubmed GEO and BioPortal databases, and was independent of KRAS mutation status. In conclusion, the central autophagy regulator TFEB is expressed and active in PDAC, but autophagy is sustained after TFEB knockdown, suggesting alternative bypass signaling. TFEB is dispensable for gemcitabine-induced cell death, but inversely correlated with KRAS expression.
\end{abstract}

Correspondence to: Dr Thilo Welsch, Department of Visceral, Thoracic and Vascular Surgery, University Hospital Carl Gustav Carus, Technische Universität Dresden, Fetscherstrasse 74, D-01307 Dresden, Germany

E-mail: thilo.welsch@uniklinikum-dresden.de

${ }^{*}$ Contributed equally

Key words: pancreatic cancer, transcription factor EB, autophagy, chemotherapy, KRAS

\section{Introduction}

Autophagy is a highly conserved self-degradation process of cellular constituents using the lysosomal machinery (1). It is physiologically induced by cellular stress or nutrient deprivation for maintenance of cell homeostasis and recycling of cellular debris. In human malignancies, including pancreatic ductal adenocarcinoma (PDAC), autophagy plays a dual, but contrary role and promotes both, tumor cell survival and death, probably depending on the tumor type and stage of tumor progress $(2,3)$. In addition to direct mechanisms of tumor growth promotion, autophagy counteracts chemotherapy-induced cell death, and autophagy inhibition sensitizes PDAC to standard chemotherapeutics such as gemcitabine $(1,4-7)$. The effectiveness of currently available chemotherapeutics against PDAC is disappointing; even in patients with resectable PDAC, the median survival is still limited to 21-24 months after curative surgery and adjuvant standard chemotherapy with gemcitabine or 5-fluorouracil (8). Therefore, a combination of chemotherapy and autophagy inhibition is intriguing, and has entered the clinical phase $(7,9)$. Clinical trials of autophagy inhibition in PDAC are further supported by the intense crosstalk of oncogenic Ras and autophagy pathways (10). Oncogenic KRAS mutations are characteristic for $>90 \%$ of all PDAC, and the link between mutation status and response to autophagy inhibition is controversially discussed $(11,12)$.

Autophagy and lysosomal gene expression are centrally orchestrated by the transcription factor EB (TFEB) $(13,14)$. Nuclear translocation and activity of TFEB is sensed and controlled by the mammalian target of rapamycin complex (mTORC1) at the cytosolic lysosome (15). Inhibition of mTORC1 or nutrient starvation abrogate TFEB phosphorylation and trigger its nuclear shift and transcription of the autophagic/lysosomal gene cascade. Interestingly, TFEB was found to be responsible for the development of a rare malignant tumor: Aberrant expression of TFEB through a specific gene translocation (Alpha-TFEB fusion) causes a distinct subtype of pediatric renal cell carcinoma (16).

This study aims to investigate the expression of the master autophagy regulator TFEB in PDAC, and its response to standard chemotherapy, to elucidate its role as a potential target for autophagy inhibition in pancreatic cancer patients. 


\section{Materials and methods}

Human pancreas samples. Specimen collection was approved by the ethics committee of the University of Heidelberg (Decision no. S-134-2010), and written informed consent was obtained from the patients. The study was performed with tissue samples obtained from patients admitted to the Department of General, Visceral and Transplantation Surgery, University Hospital Heidelberg, for surgical treatment of PDAC or benign disease. Diagnoses were established by a pathologist according to the World Health Organization (WHO) classification.

Cell lines, reagents, and antibodies. The human pancreatic cancer cell lines PANC-1, MIA PaCa-2, and BxPC-3 were purchased from ATCC (LGC Standards, Wesel, Germany). They were cultured in a humidified atmosphere containing $5 \% \mathrm{CO}_{2}$ at $37^{\circ} \mathrm{C}$. RPMI-1640 supplemented with $10 \%$ fetal calf serum (FCS) was used as the medium for PANC-1. MIA PaCa- 2 cells were cultured in DMEM, with $10 \%$ FCS and $2.5 \%$ horse serum added.

The following antibodies were used: mouse anti-KRAS (sc-30, Santa Cruz Biotechnology, Heidelberg, Germany), rabbit anti-LC3A/B (\#4108, Cell Signaling, Danvers, MA, USA), rabbit anti-Akt (\#9272, Cell Signaling), rabbit antiphospho-AKT (Ser473, \#9271, Cell Signaling), rabbit anti-TFEB (\#4240, Cell Signaling), anti-TFEB (V-17, Santa Cruz Biotechnology), mouse monoclonal anti-TFEB (clone 3E1-G6, Sigma-Aldrich, Taufkirchen, Germany), antiPARP (\#9542, Cell Signaling), and rabbit anti-GAPDH (\#2118, Cell Signaling).

The chemotherapeutic agent gemcitabine (Hexal, Holzkirchen, Germany) was added to the cells after their treatment with siRNAs in indicated concentrations, and incubated for 48-72 h. Choloroquine (chloroquine disphophate salt, Sigma-Aldrich) was diluted in $\mathrm{H}_{2} \mathrm{O}$ to the indicated concentrations.

Transfection and siRNA. The plasmid ptfLC3 was purchased from Addgene (\#21074, Cambridge, MA, USA). It encodes for LC3 tagged with mRFP and EGFP to distinguish between autophagosomes and autophagolysosomes based on its loss of GFP in lysosomal acidic environment (17). For the transfection, Lipofectamine 2000 (Life Technologies, Darmstadt, Germany) was used according to the manufacturer's protocol.

Transfection of siRNAs was performed using HiPerfect Transfection reagent (Qiagen, Hilden, Germany) according to the manufacturer's protocol. The following sequences were targeted by siRNAs: KRAS 5'-GGCTATATTTACATGCTA CTA-3' [Eurofins MWG Operon, Ebersberg, Germany (18)], TFEB 5'-CACAACTTAATTGAAAGGAGA-3' (Qiagen), and KIF11 (Eg5) 5'-AACTGAAGACCTGAAGACAAT-3' as the positive control (19); and nonsense siRNA (Allstars, Qiagen) served as the negative control. All transfections were performed with a concentration of $30 \mathrm{nM}$ per target gene and an incubation time of 48-72 $\mathrm{h}$.

Quantitative RT-PCR. RNA was isolated with the Nucleo Spin II kit (Macherey-Nagel, Düren, Germany) and converted to cDNA with the High Capacity cDNA Reverse Transcription kit (Applied Biosystems, Foster City, CA, USA) according to the manufacturers' protocols. The quantitative RT-PCR was performed using the Power SYBR Green Master Mix (Life Technologies). ACTB served as a housekeeping gene for normalization of $\mathrm{C}_{\mathrm{t}}$-values. The Delta-Delta- $\mathrm{C}_{\mathrm{t}}$-method was used to quantify gene expression levels. The specific TFEB primers were obtained from Search-LC (Heidelberg, Germany). RT-PCR results in human tissues are presented as TFEB transcripts per 10,000 cyclophilin B copies (10 kCPB).

Western blotting. Cells were washed and lysed on ice by RIPA buffer containing protease and phosphatase inhibitors (Thermo Fisher Scientific, Rockford, IL, USA). For extracting proteins, cell lysates were centrifuged for $15 \mathrm{~min}$ at $11,000 \mathrm{x}$ g followed by concentration measurement of the supernatant with the Pierce BCA Protein assay kit (Thermo Fisher Scientific). A total of 10-15 $\mu \mathrm{g}$ protein per sample was then mixed with 4X LDS sample buffer and Sample Reducing Agent (Thermo Fisher Scientific) and heated to $90^{\circ} \mathrm{C}$ for $10 \mathrm{~min}$ with gentle agitation according to the manufacturer's protocol of the NuPAGE SDS-PAGE Gel system (Thermo Fisher Scientific). Probes were separated electrophoretically in a 4-12\% Bis-Tris NuPAGE Gel (Thermo Fisher Scientific) and blotted onto a nitrocellulose membrane using the semidry blot NuPAGE system. After blotting, the membrane was blocked for $1 \mathrm{~h}$ at room temperature in a blocking solution, containing 5\% Slim Fast powder (Allpharm Vertriebs GmbH, Messel, Germany) dissolved in TBS-Tween $0.1 \%$ buffer. The blots were then incubated with primary antibodies (anti-Akt 1:1,000, anti-GAPDH 1:5,000, anti-KRAS 1:100, anti-LC3A/B 1:500, antiphospho-Akt 1:1,000, anti-TFEB 1:500) in a blocking solution overnight at $4^{\circ} \mathrm{C}$. After three washing steps with TBS-Tween $0.1 \%$ buffer, the membrane was incubated with HRP-tagged, host-specific secondary antibodies (Cell Signaling) for $1 \mathrm{~h}$ at room temperature. After three additional washing steps, chemiluminescence solution (Immobilon Western Chemi, Millipore, Billerica, MA, USA) was added for detection of protein bands using G:Box Chemi XT4 (Syngene, Cambridge, UK). GAPDH served as the loading control. Quantification of protein intensity was performed using ImageJ (Version 2.0.0; http://imagej.net/).

Immunhistochemistry. The paraffin-embedded tissue sections were immunostained with horseradish peroxidase (HRP) labeled polymer (Dako Deuschland $\mathrm{GmbH}$, Hamburg, Germany). Shortly, consecutive tissue sections were deparaffinized and rehydrated in progressively decreasing concentrations of ethanol. After the antigens were retrieved by boiling the tissue sections in $10 \mathrm{mM}$ citrate buffer for $3 \times 5 \mathrm{~min}$ in the microwave oven, endogenous peroxidase activity was blocked with $3 \%$ hydrogen peroxide in methanol for $10 \mathrm{~min}$. The slides were then placed in a washing buffer $(10 \mathrm{mM}$ Tris- $\mathrm{HCl}, 0.85 \% \mathrm{NaCl}, 0.1 \%$ bovine serum albumin, $\mathrm{pH} 7.4$ ), and the sections were incubated overnight at $4^{\circ} \mathrm{C}$ with the rabbit anti-TFEB antibody (\#4240, Cell Signaling) diluted in antibody diluent (Dako) or rabbit immunoglobulin as negative control (Dako). Then, the slides were incubated with a secondary anti-rabbit-labeled polymer (Dako) for $45 \mathrm{~min}$ at room temperature. The immunoreactivity was visualized using a 3,3'-diaminobenzidine (DAB) chromogen $/ \mathrm{H}_{2} \mathrm{O}_{2}$ system (Dako), and the sections were counterstained with Mayer's 

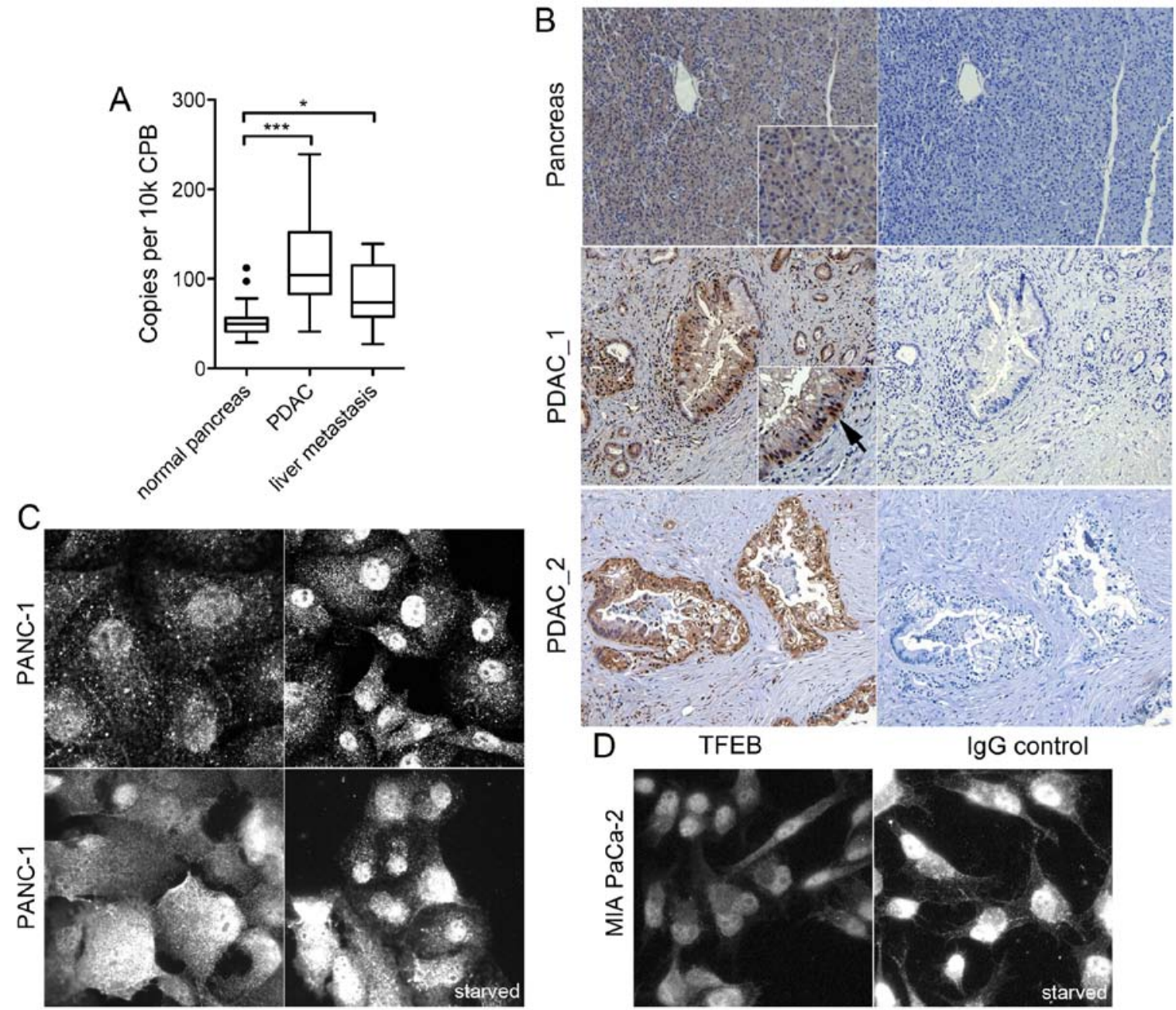

Figure 1. Expression analysis of TFEB in human PDAC samples and cell lines. (A) Quantitative mRNA expression in human normal pancreas (n=18), PDAC $(\mathrm{n}=45)$, and PDAC liver metastasis $(\mathrm{n}=8)$. Data were normalized to cyclophilin $\mathrm{B}(\mathrm{CPB})$ and presented as box plot (median, interquartile range). ${ }^{* * *} \mathrm{P}<0.0001$; ${ }^{* *} \mathrm{P}<0.01$; ${ }^{*} \mathrm{P}<0.05$. (B) Representative immunohistochemistry analysis of TFEB in normal pancreas, and in two PDAC samples (PDAC_1 and PDAC_2) next to the respective immunoglobulin $\mathrm{G}$ control (right). The arrow points to the nuclear staining. Magnification was x 200. (C and D) Immunofluorescence analysis of TFEB in PANC-1 (C) and MIA PaCa-2 cells (D) under normal serum conditions, or under serum deprivation for $24 \mathrm{~h}$ (starved). Immunoreactivity was tested with three different antibodies: goat polyclonal anti-TFEB V-17 [(C), upper left)], mouse monoclonal anti-TFEB clone 3E1-G6 [(C), upper right], and rabbit anti-TFEB \#4240 [(C), lower row; (D)]. The image width represents $100 \mu \mathrm{m}$ except the upper left image in (C) $(80 \mu \mathrm{m})$.

hematoxylin. Finally, the slides were viewed with an Axiophot microscope (Carl Zeiss, Göttingen, Germany) coupled with a charge-coupled device (CCD) camera (AxioVision, Zeiss, Jena, Germany).

Immunofluorescence staining and confocal imaging. Cells were grown on collagen-A $(0.1 \mathrm{mg} / \mathrm{ml})$ coated glass cover slips and treated with serum starvation or siRNA transfection. Immunostaining was performed as described previously (20). Briefly, cells were fixed with $2 \%$ paraformaldehyde, permeabilized by $0.3 \%$ Triton X-100 for $5 \mathrm{~min}$, and incubated in a blocking solution ( $2 \%$ fetal calf serum, $2 \%$ bovine serum albumin, $0.2 \%$ fish gelatine resolved in PBS). Samples were then incubated with the primary antibody for $1 \mathrm{~h}$. After several washing steps with PBS, cover slips were treated with Cy3- or Alexa Fluor 488-tagged secondary antibodies. Cell nuclei were stained with DAPI (1:200). Confocal imaging was performed on a Leica SP5 laser-scanning microscope using the 63x oil-immersion objectives (Leica Microsystems, Heidelberg, Germany). The fluorescence intensity of TFEB was quantified by calculating the ratio of the mean intensity in the nucleus and cytoplasm of the respective cells using ImageJ (Version 2.0.0; http://imagej.net/).

Apoptosis assay and cell count. Cells including the supernatant were collected in a FACS tube. After centrifugation for $5 \mathrm{~min}$ at $4^{\circ} \mathrm{C}$ and $1,500 \mathrm{rpm}$, the supernatant was discarded and pellets were used for propidium iodide (PI)/Annexin V apoptosis assays. For the apoptosis assay, the samples were washed twice with FACS binding buffer (10 mM HEPES $\left.\mathrm{pH} 7.4,2.5 \mathrm{mM} \mathrm{CaCl}_{2}, 140 \mathrm{nM} \mathrm{NaCl}\right)$, and PI $(50 \mu \mathrm{g} / \mathrm{ml})$ (Sigma-Aldrich) and Annexin V (BD Biosciences, Heidelberg, Germany) were added. Measurements were performed with FACSCalibur (BD Biosciences) and analyzed using WinMDI (Scripps Research Institute, La Jolla, CA, USA). Apoptotic cells were defined as the sum of $\mathrm{PI}^{+} / \mathrm{Annexin}^{+}$(late apoptosis) and $\mathrm{PI}^{-} / A n n e x i n \mathrm{~V}^{+}$(early apoptosis) cells.

The relative cell count was determined, which reflects proliferation inhibition and apoptosis. The treated cells were harvested by trypsinization and counted with the TC-20 


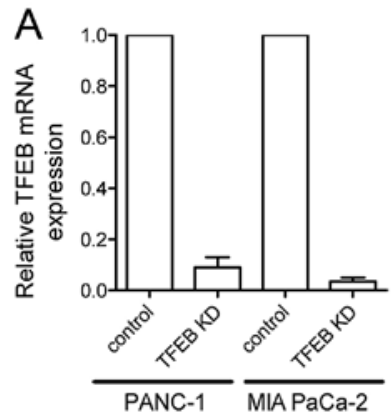

C

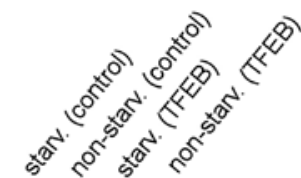

TFEB

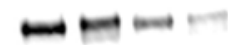

LC3

GAPDH

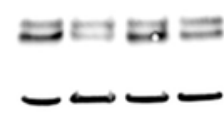

E

PANC-1
D

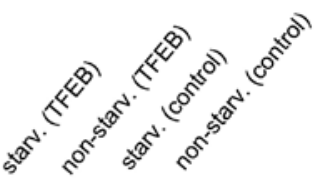

TFEB

LC3

GAPDH

MIA PaCa-2
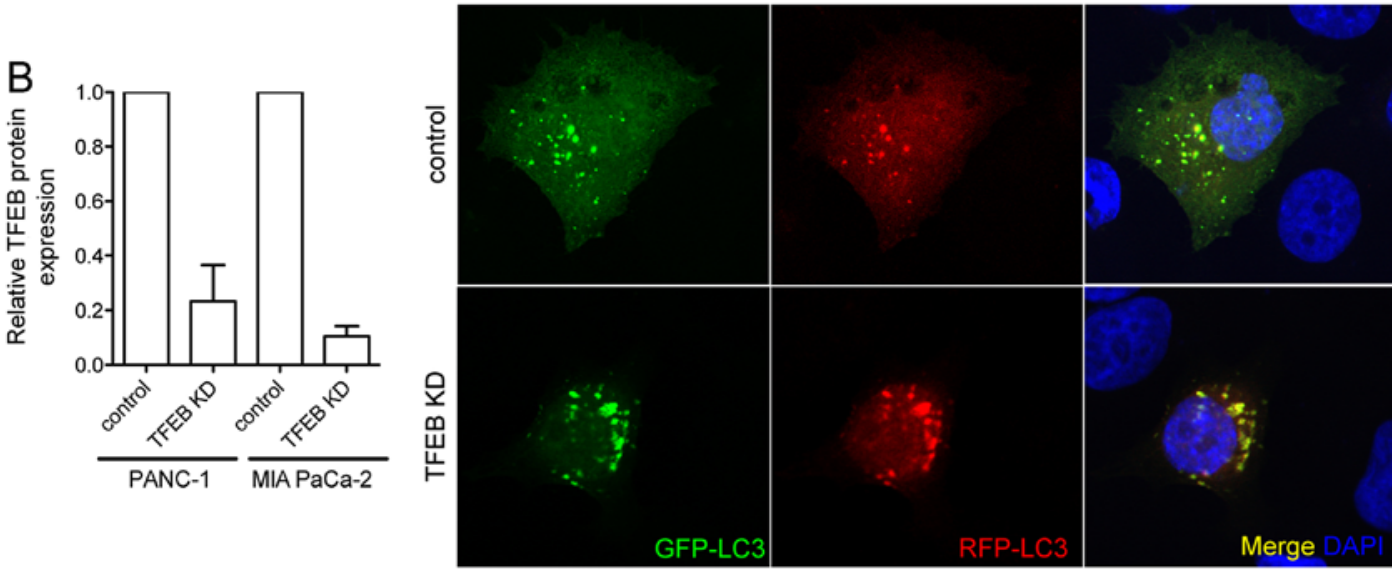

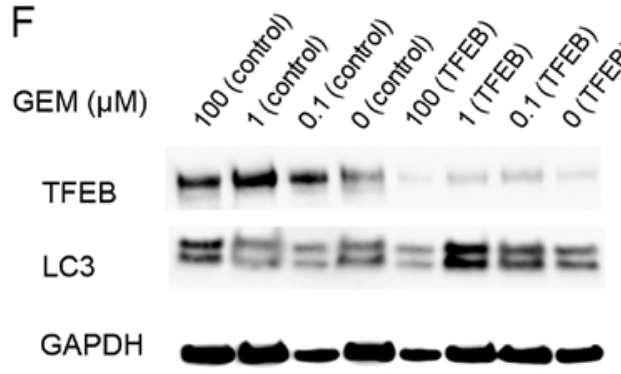

PANC-1

G GEM $(\mu M)$

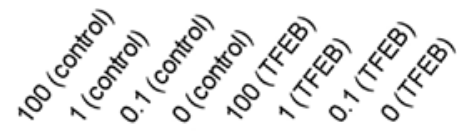

TFEB

LC3

GAPDH

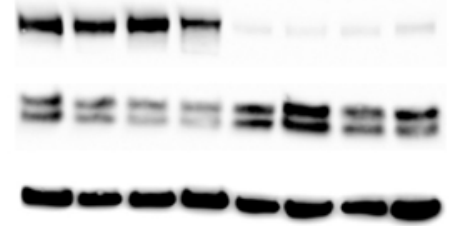

MIA PaCa-2

Figure 2. TFEB silencing and autophagy regulation in PDAC cells. (A) Quantitative mRNA analysis of TFEB expression after specific siRNA knockdown (48 h) in PANC-1 and MIA PaCa-2 cells. (B) Quantitation of TFEB protein expression after siRNA knockdown (48 h) normalized to GAPDH expression. Experiments were performed as triplicates; data are plotted as mean \pm SEM. (C and D) Western blotting of LC3-I (LC3, upper lane) and -II (LC3, lower lane) after TFEB siRNA knockdown (TFEB) and under serum deprivation for $24 \mathrm{~h}$ (starved) in PANC-1 (C) and MIA PaCa-2 cells (D). GAPDH served as the loading control. (E) Immunofluorescence analysis of PANC-1 cells transiently expressing the mRFP-EGFP tandem tagged LC3 plasmid (ptfLC3). TFEB knockdown (KD) did not significantly affect the ratio of autophago (EGFP+mRFP-positive)- to autophagolysosomes (mRFP-positive). Image width represents $60 \mu \mathrm{m}$. (F and G) Western blot analysis of TFEB and LC3-I and -II after treatment with gemcitabine for $48 \mathrm{~h}$ using ascending dosages in PANC-1 (F) and MIA PaCa-2 (G) cells.

Automated Cell Counter (Bio-Rad, München, Germany). Cell numbers were normalized to the media controls.

Statistical analysis. If not indicated otherwise, data are presented as mean \pm standard error of the mean (SEM) and the two-sided, unpaired t-test was used as the statistical test. Results were analyzed and plotted using the Prism 5 software (GraphPad Software, La Jolla, CA, USA).

\section{Results}

Expression analysis of TFEB in human pancreas and PDAC. The expression of TFEB transcription was analyzed in human normal pancreas tissue, in PDAC samples and in liver metas- tases. The expression levels in primary tumor samples $(n=45)$ were significantly elevated compared with normal pancreas tissue $(\mathrm{P}<0.001)$, and PDAC liver metastasis had also higher TFEB expression compared with normal pancreas $(\mathrm{P}=0.02$, Fig. 1A). Immunohistochemistry revealed a weak to moderate cytoplasmic TFEB expression in normal ductal cells, but no nuclear staining; moderate or high TFEB staining and nuclear localization was found in 11 of 15 cancer specimens $(\mathrm{P}=0.02$; Fisher's exact test) (Fig. 1B).

Likewise, TFEB expression was investigated in human PDAC cell lines. Immunoreactivity was compared using three different, specific antibodies directed against TFEB. The primary PDAC cell lines PANC-1 and MIA PaCa-2 showed both cytoplasmic and nuclear TFEB expression under 

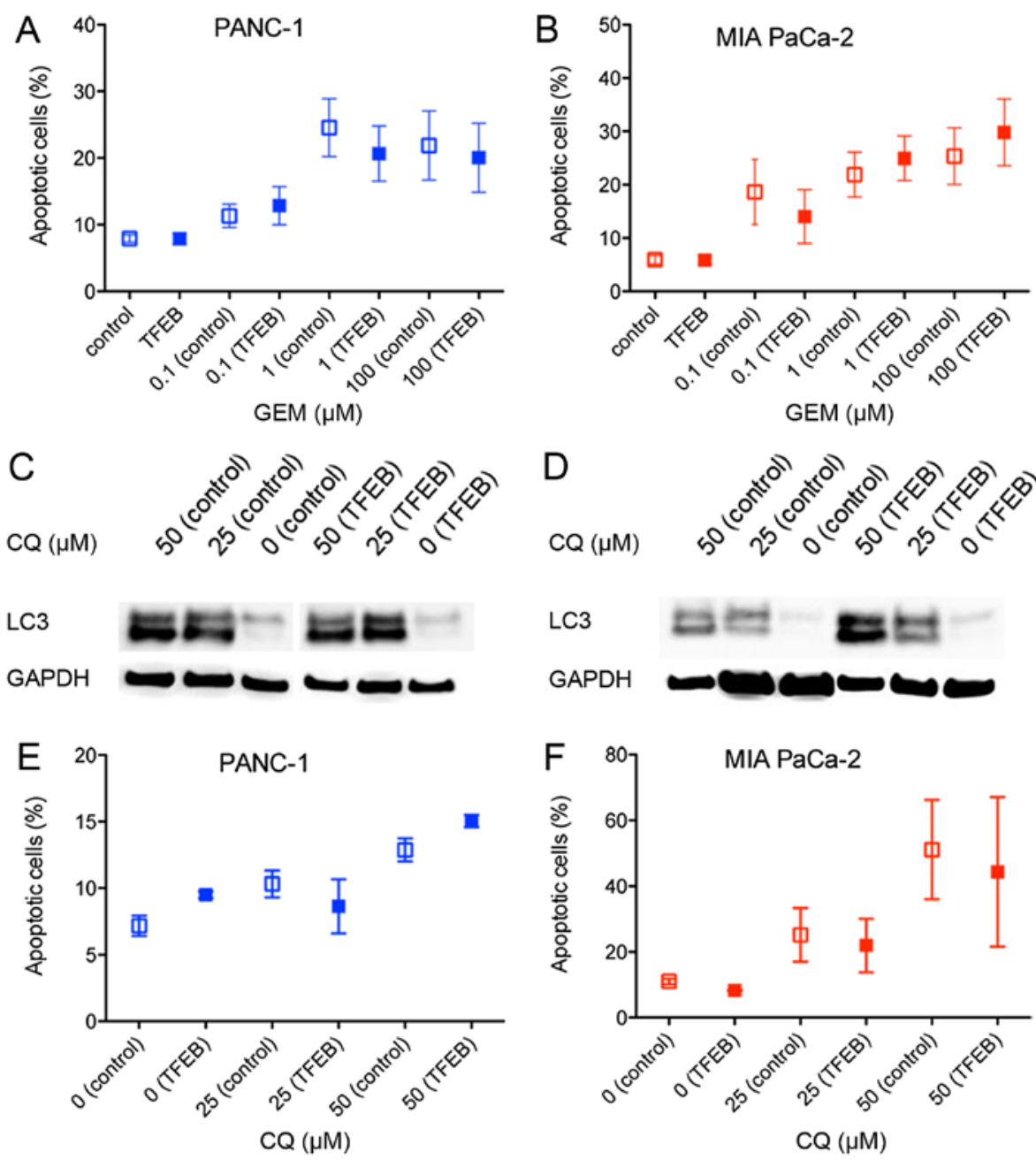

Figure 3. Treatment response in PDAC cells with respect to TFEB expression. (A and B) A transient siRNA knockdown of TFEB was established in PANC-1 (A) and MIA PaCa-2 (B) cells, and the cells were treated with ascending dosages of gemcitabine (GEM) for $48 \mathrm{~h}$. Apoptotic cells were measured using the PI/Annexin V assay, and data were plotted as mean \pm SEM. (C and D) PANC-1 (C) and MIA PaCa-2 (D) cells were exposed to chloroquine (CQ) at ascending dosages, and LC-3 expression was analyzed by western blotting with (TFEB) and without (control) siRNA knockdown of TFEB. (E and F) Percentage of apoptotic cells after treatment with CQ at different dosages (mean \pm SEM). TFEB expression was transiently silenced in the cells as indicated (TFEB).

normal culture conditions. Serum starvation led to minor enhancement of the nuclear staining (Fig. 1C and D). The ratio of nucleus and cytoplasmic fluorescence intensity was significantly increased by starvation $(\mathrm{P}=0.01, \mathrm{n}=35$ cells $)$. These results indicate TFEB activation in PDAC cells even under basal conditions and functional sensing of the available nutrient supply.

Autophagy dependence on TFEB in PDAC cells. To assess whether TFEB expression affected autophagy in PDAC cells, a transient TFEB RNA interference (siRNA) was established, which achieved a mean protein downregulation to 23 and $11 \%$ in PANC-1 and MIA PaCa-2 cells, respectively (Fig. 2A and B). Both PDAC cell lines responded to starvation with increased expression of TFEB and microtubule-associated protein 1A/1B-light chain 3 (LC3)-II. The starvation-induced upregulation of LC3-II was even maintained during TFEB silencing (Fig. 2C and D). We further explored whether TFEB silencing affected the autophagy route from autophago- to autophagolysosomes. To this end, a red fluorescent proteingreen fluorescent protein (RFP-GFP) tandem tagged LC3 protein was exogenously expressed to differentiate autophagosomes (GFP and RFP-positive) from autophagolysosomes (GFP-negative, but RFP-positive) (17). However, there was neither a measurable difference in the LC3-positive vesicle number, nor in the ratio of autophago- to autophagolysosomes after TFEB silencing (Fig. 2E). Consequently, TFEB is an indicator of autophagy in PDAC cells, but low expression levels are sufficient to run the autophago-to-lysosome machinery. This was further supported by chemotherapeutic treatment of the cancer cells with gemcitabine, which caused a nearly dose-dependent LC3-II accumulation using gemcitabine at concentrations $\leq 100 \mu \mathrm{M}$, indicating increased autophagy, regardless of the amount of TFEB protein (Fig. 2F and G). Thus, the autophagy response of PDAC cells to gemcitabine did not solely depend on the TFEB expression levels.

Chemotherapy-induced cytotoxicity. Previous data have demonstrated that autophagy sustained PDAC cell survival and counteracted chemotherapeutic cytotoxicity, but it is unknown whether these effects are mediated by TFEB $(5,7)$. Therefore, 
A

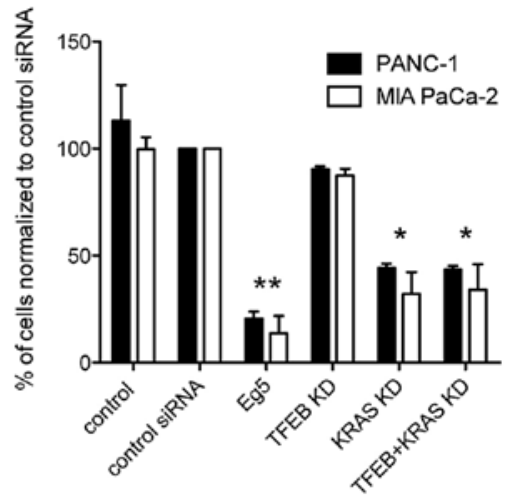

C

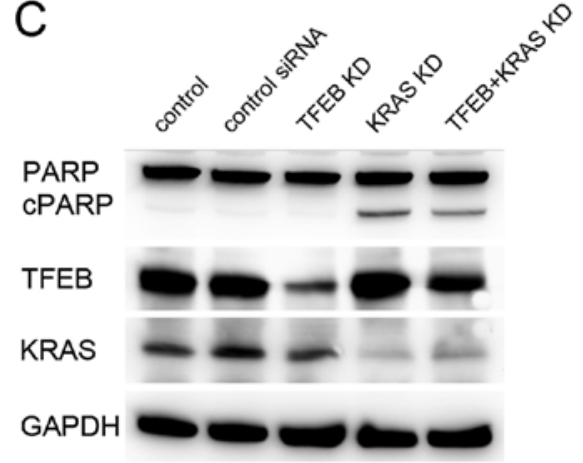

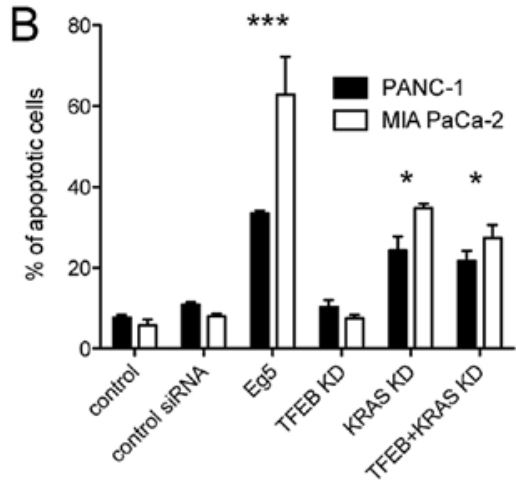

D

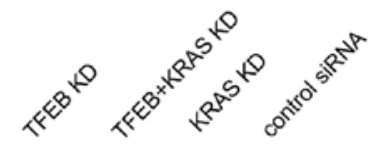

LC3

Akt

pAkt

GAPDH
$E$

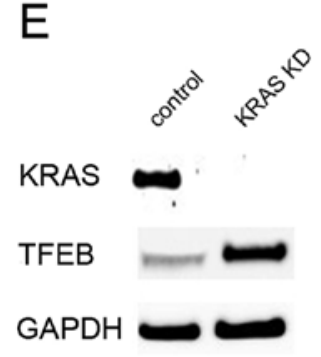

$\mathrm{F}$

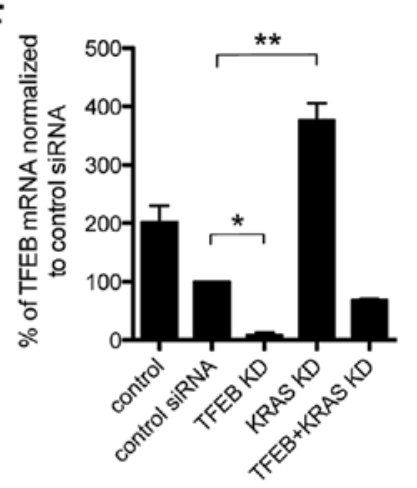

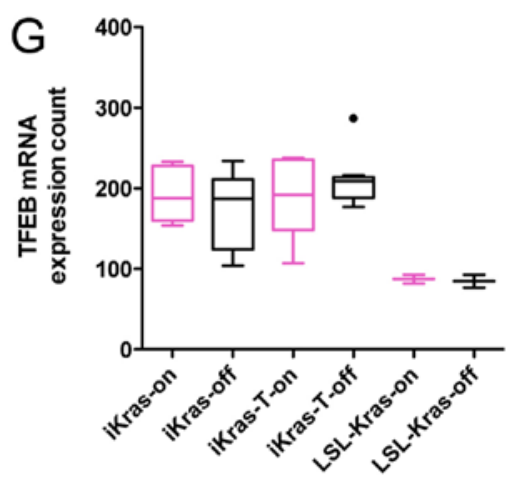
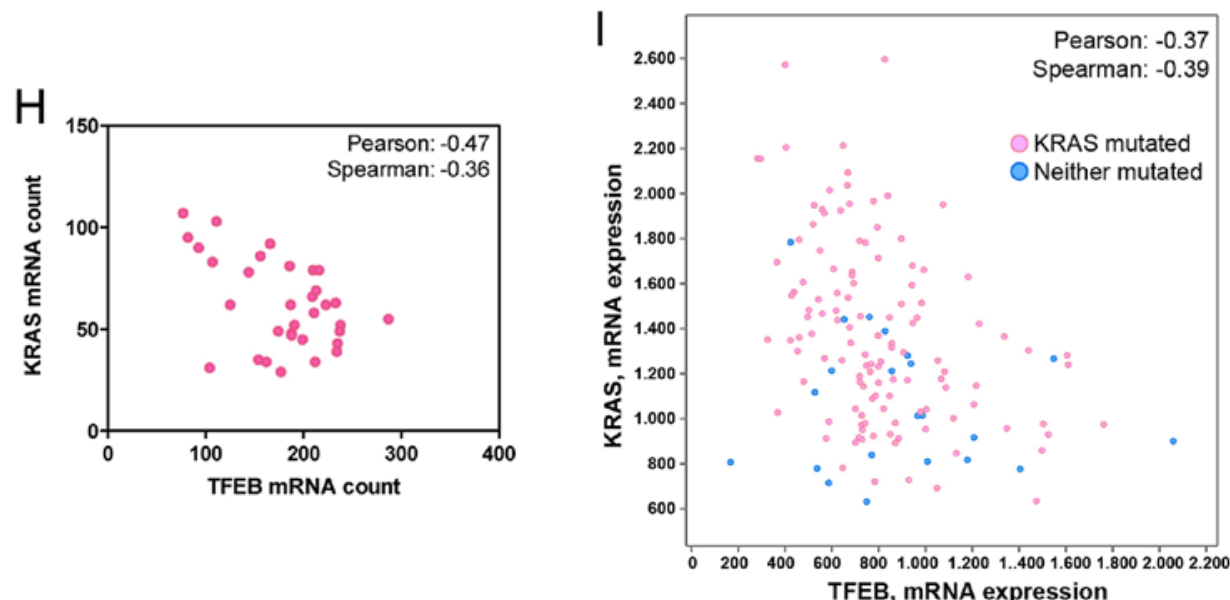

Figure 4. Correlation of KRAS and TFEB expression in pancreatic cancer cells. (A and B) PDAC cell count (A) and apoptosis (B) after siRNA knockdown (KD) of Eg5, TFEB, KRAS or simultaneous TFEB, and KRAS KD for $72 \mathrm{~h}$. Experiments were performed as triplicates (mean \pm SEM). (C) Western blotting of indicated proteins in PANC-1 cells after TFEB and KRAS KD for $72 \mathrm{~h}$. Cleaved PARP (cPARP) indicates apoptosis after KRAS KD. (D) Western blotting of LC3-I and -II after TFEB and KRAS KD. KRAS KD was not accompanied by increased phospho-AKT (pAkt) in PANC-1 cells. (E) KRAS KD for $48 \mathrm{~h}$ led to increased TFEB protein expression (PANC-1 cells). (F) Quantitative mRNA analysis after TFEB and KRAS KD in PANC-1 cells (mean \pm SEM). (G) TFEB expression analysis using the data set record GDS4343 (NCBI Gene Expression Omnibus Data Set). Cultured parenteral iKras p53L/+ PDAC cells (iKras, n=10), the respective xenograft tumors (iKras-T, $n=19$ ), and control cells (LSL-iKras, $n=4$ ) expressed doxycycline-dependent oncogenic KRAS G12D (on). KRAS expression was significantly elevated in LSL-iKras cells compared with the iKras and iKras-T cells (26). Data are presented as a box plot. (H) Correlation of TFEB and KRAS mRNA expression in the iKras parenteral, xenograft, and control cells ( $\mathrm{n}=33$ ). (I) Correlation of TFEB and KRAS expression in human PDAC samples using the cBioPortal database (http://www.cbioportal.org/; pancreatic adenocarcinoma, 145 samples included). Pink dots represent tumor samples with KRAS mutations. ${ }^{* * *} \mathrm{P}<0.0001 ;{ }^{* *} \mathrm{P}<0.01$; ${ }^{*} \mathrm{P}<0.05$. 
cell death (apoptosis) induced by gemcitabine treatment was examined after TFEB siRNA depletion. Only 20-30\% of the cancer cells went into apoptosis at concentrations $>1 \mu \mathrm{M}$ gemcitabine, and there was no significant difference depending on the TFEB expression level (Fig. 3A and B). Because it has been shown that the known inhibitor of autophagosome degradation, chloroquine (CQ), can suppress PDAC growth, we further explored whether the cytotoxic effect of CQ depends on TFEB activity. Autophagy inhibition by CQ was seen in both cell lines (Fig. 3C and D). Induction of apoptosis was found in $15-50 \%$ of the cells using established concentrations of CQ, but there was no difference in the apoptosis rate when TFEB expression was silenced (Fig. 3E and F).

KRAS and TFEB expression - a link between driver mutation and autophagy regulation. Because the results indicated that autophagy sustained tumor cell survival in PDAC, we further investigated the crosstalk between the PDAC driver oncogene $K R A S$ and autophagy. Ras signaling can promote, but also inhibit, autophagy through use of different cascades including Rac1, Raf1, and phosphoinositide-3-kinase (PI3K) signaling (10). The latter PI3K downsignaling involves the Akt/mTOR1 pathway, and can negatively regulate autophagy activity (21). Silencing of KRAS expression increased cell death and apoptosis of the cancer cells independently of TFEB expression, and TFEB knockdown had no significant effect on cell death (Fig. 4A-C). Blockage of KRAS expression led to an unchanged or minor increase in the LC3-II fraction, which was not accompanied by changes in Akt phosphorylation (Fig. 4D). However, KRAS silencing resulted in reciprocally augmented levels of TFEB protein and transcripts (Fig. 4E and F), whereas TFEB knockdown did not enhance KRAS expression. If oncogenic KRAS activity instead of KRAS transcription levels would determine TFEB expression, wild-type PDAC cells (e.g., BxPC-3) were expected to harbor higher TFEB concentrations. However, TFEB expression was comparably low in the KRAS wild-type BxPC-3 cells (data not shown). These findings were supported by analysis of published data of an inducible KRAS mouse model; oncogenic $K R A S$ activity status (on/off) did not influence TFEB expression, but KRAS expression levels negatively and significantly correlated with TFEB expression (Fig. 4G and H). Moreover, the inverse relationship of KRAS and TFEB expression levels could be confirmed in human PDAC samples, and again, there was no TFEB dependence on the KRAS mutation status (Fig. 4I).

\section{Discussion}

Although autophagy can synergize with chemotherapy in other types of cancer, accumulating evidence points to the fact that autophagy is required for or supports PDAC growth $(5,7,22)$. In our previous studies, we could demonstrate large lysosomal compartments and oncogene-associated, autophagy-mediated pathways in metastasized PDAC cells (20). For these reasons, targeting autophagy has grown to an important field in PDAC research. This study explored the central autophagy regulator TFEB in PDAC, and its role for autophagy and tumor cell survival. TFEB belongs to the microphtalmia MiT/TFE family of transcription factors (MITF, TFE3, TFEB and TFEC)
(23), and this study demonstrated its elevated expression and nuclear localization in human PDAC cells. While this report was under preparation, Perera et al published a comprehensive analysis of the MiT/TFE proteins in PDAC (24). These data support both, TFEB expression in PDAC, and the constitutive nuclear translocation in these cells. The authors further found that the MiT/TFE proteins are decoupled from regulatory control mechanisms and are required to maintain the intracellular amino acid pool (24). In contrast to this study, they found the MiT/TFE proteins to be critical for autophagy-lysosome function, and that knockdown impaired primary PDAC cell growth. Moreover, knockdown of TFE3 and MITF abolished xenograft tumor growth of PANC-1 cells. These discrepant results might be explained by the relative high expression of TFE3 in PDAC and PANC-1 cells compared with TFEB (24), which may compensate for autophagy biogenesis after TFEB silencing. Both studies strongly indicate nuclear expression of TFE3 (24) or TFEB (this study) in PDAC, and therefore conflict with an immunohistochemistry analysis of a subset of 56 PDAC samples, which lacked significant nuclear staining (16). Hence, one can speculate whether nuclear TFE3/TFEB positivity depends on a different antibody affinity in the studies or on the biological tumor stage.

These data also show that chemotherapeutic treatment with gemcitabine induces autophagy, and that autophagy inhibition with chloroquine increases tumor cell apoptosis; both results are in line with a potentiated PDAC cell response to simultaneous chemotherapy and chloroquine treatment (5). However, PDAC cells may use other central autophagy regulators than TFEB for chemotherapy-induced autophagy, as TFEB knockdown did not impair autophagy induction.

In fact, promotion of tumorigenesis by autophagy appears to be an outstanding hallmark of PDAC, and was linked to oncogenic KRAS activity $(3,12)$. Oncogenic KRAS is the signature mutation of PDAC and orchestrates not only altered metabolism of glucose and glutamine $(25,26)$, but also promotes macropinocytosis and makes the tumor cells (namely, their mitochondrial respiration) dependent on autophagic flux; defective autophagosome formation causes accumulation of abnormal mitochondria and reduced oxygen consumption (7,27-30). In contrast, oncogenic KRAS activity can impair autophagy through the PI3K/Akt/mTORC1 signaling pathway (10). Activated mTORC1 retains the cytoplasmic TFEB pool and inhibits its nuclear activity (15), but it had been unknown whether TFEB is critical for KRASmediated autophagy regulation. These results demonstrate that PANC-1 and MIA PaCa-2 cell growth is sustained on KRAS expression, and KRAS inhibition, but not TFEB inhibition, increased apoptosis. This is in good agreement with our published results of simultaneous gene silencing of KRAS and apoptotic genes (18). Both primary PDAC cell lines (PANC-1, MIA PaCa-2) express activating mutations of KRAS and inactivating mutations of the P53 gene (31). Although under debate, recent experiments provided evidence that autophagy fuels PDAC growth regardless of a simultaneous P53 inactivation (3), which is supported by the data that demonstrated impaired growth of both PDAC cell lines with inactivated P53 status after autophagy inhibition. Because of the high basal autophagic flux in PDAC cells, pro-autophagy signaling by KRAS should outweigh its inhibitory effect on autophagy 
(e.g., through the PI3K/Akt/mTORC1 pathway). Thus, KRAS knockdown did not significantly augment autophagy, and the phosphorylation status of Akt remained unchanged. One can hypothesize that increased TFEB expression compensates for depleted KRAS, to maintain autophagy in PDAC cells. The inverse correlation of KRAS and TFEB expression in PDAC cells was a novel finding and was confirmed in a mouse model and in human PDAC samples. The diverging effects of KRAS gene silencing and oncogenic KRAS activity in PDAC may suggest that KRAS and TFEB are interrelated in a way that loss of oncogenic KRAS levels elicits enhanced TFEB transcription for promotion of pro-tumorigenic, autophagic flux and amino acid supply (24).

This study has some drawbacks and limitations. For example, TFEB expression was not completely depleted in the cell lines, leaving a basal gene expression, and the presented results were not confirmed by additional siRNA sets; other members of the MiT/TFE protein family were not examined, and the effect of increased TFEB expression after KRAS knockdown on the tumor cells was not further elucidated. However, the results strongly point to the fact that PDAC cells require TFEB, but can compensate for TFEB depletion to maintain tumorigenic autophagy. Although PDAC growth and metabolism is tailored to and depends on autophagy, and TFEB centrally orchestrates cellular lysosomal and autophagic programs, targeting TFEB appears not to be a promising anticancer strategy for PDAC. Based on these findings, further studies will focus on how TFEB and the associated MiT/TFE proteins are regulated by the key oncogene $K R A S$.

\section{Acknowledgements}

This study was supported by a German Research Foundation (DFG) research grant to T.W. (WE 4816/1-2). Pancreatic samples were obtained from Pancobank (Professor Büchler, Dr Giese, E. Soyka, M. Stauch) supported by the BMBF (BMBF grant 01EY1101 to Professor Schirmacher), Heidelberger Stiftung Chirurgie, and the BMBF grants 01GS08114 and 01ZX1305C.

\section{References}

1. Galluzzi L, Pietrocola F, Bravo-San Pedro JM, Amaravadi RK, Baehrecke EH, Cecconi F, Codogno P, Debnath J, Gewirtz DA, Karantza V, et al: Autophagy in malignant transformation and cancer progression. EMBO J 34: 856-880, 2015.

2. Ropolo A, Bagnes CI, Molejon MI, Lo Re A, Boggio V, Gonzalez CD and Vaccaro MI: Chemotherapy and autophagymediated cell death in pancreatic cancer cells. Pancreatology 12 : 1-7, 2012.

3. Yang A and Kimmelman AC: Inhibition of autophagy attenuates pancreatic cancer growth independent of TP53/TRP53 status. Autophagy 10: 1683-1684, 2014.

4. Donohue E, Thomas A, Maurer N, Manisali I, ZeisserLabouebe M,Zisman N, Anderson HJ, Ng SS, Webb M, Bally M, et al: The autophagy inhibitor verteporfin moderately enhances the antitumor activity of gemcitabine in a pancreatic ductal adenocarcinoma model. J Cancer 4: 585-596, 2013.

5. Hashimoto D, Bläuer M, Hirota M, Ikonen NH, Sand J and Laukkarinen J: Autophagy is needed for the growth of pancreatic adenocarcinoma and has a cytoprotective effect against anticancer drugs. Eur J Cancer 50: 1382-1390, 2014.

6. Papademetrio DL, Cavaliere V, Simunovich T, Costantino S, Campos MD, Lombardo T, Kaiser CM and Alvarez E: Interplay between autophagy and apoptosis in pancreatic tumors in response to gemcitabine. Target Oncol 9: 123-134, 2014
7. Yang S, Wang X, Contino G, Liesa M, Sahin E, Ying H, Bause A, Li Y, Stommel JM, Dell'antonio G, et al: Pancreatic cancers require autophagy for tumor growth. Genes Dev 25: 717-729, 2011.

8. Neoptolemos JP, Stocken DD, Bassi C, Ghaneh P, Cunningham D, Goldstein D, Padbury R, Moore MJ, Gallinger S, Mariette C, et al; European Study Group for Pancreatic Cancer: Adjuvant chemotherapy with fluorouracil plus folinic acid vs gemcitabine following pancreatic cancer resection: A randomized controlled trial. JAMA 304: 1073-1081, 2010.

9. Wolpin BM, Rubinson DA, Wang X, Chan JA, Cleary JM, Enzinger PC, Fuchs CS, McCleary NJ, Meyerhardt JA, Ng K, et al: Phase II and pharmacodynamic study of autophagy inhibition using hydroxychloroquine in patients with metastatic pancreatic adenocarcinoma. Oncologist 19: 637-638, 2014.

10. Schmukler E, Kloog Y and Pinkas-Kramarski R: Ras and autophagy in cancer development and therapy. Oncotarget 5: 577-586, 2014.

11. Eser S, Schnieke A, Schneider G and Saur D: Oncogenic KRAS signalling in pancreatic cancer. Br J Cancer 111: 817-822, 2014.

12. Morgan MJ, Gamez G, Menke C, Hernandez A, Thorburn J, Gidan F, Staskiewicz L, Morgan S, Cummings C, Maycotte P, et al: Regulation of autophagy and chloroquine sensitivity by oncogenic RAS in vitro is context-dependent. Autophagy 10: 1814-1826, 2014.

13. Settembre $C$ and Ballabio A: TFEB regulates autophagy: An integrated coordination of cellular degradation and recycling processes. Autophagy 7: 1379-1381, 2011.

14. Settembre C, Di Malta C, Polito VA, Garcia Arencibia M, Vetrini F, Erdin S, Erdin SU, Huynh T, Medina D, Colella P, et al: TFEB links autophagy to lysosomal biogenesis. Science 332: 1429-1433, 2011.

15. Settembre C, Zoncu R, Medina DL, Vetrini F, Erdin S, Erdin S, Huynh T, Ferron M, Karsenty G, Vellard MC, et al: A lysosometo-nucleus signalling mechanism senses and regulates the lysosome via mTOR and TFEB. EMBO J 31: 1095-1108, 2012.

16. Argani P, Laé M, Hutchinson B, Reuter VE, Collins MH, Perentesis J, Tomaszewski JE, Brooks JS, Acs G, Bridge JA, et al: Renal carcinomas with the $t(6 ; 11)(\mathrm{p} 21 ; \mathrm{q} 12)$ : Clinicopathologic features and demonstration of the specific alpha-TFEB gene fusion by immunohistochemistry, RT-PCR, and DNA PCR. Am J Surg Pathol 29: 230-240, 2005.

17. Kimura S, Noda T and Yoshimori T: Dissection of the autophagosome maturation process by a novel reporter protein, tandem fluorescent-tagged LC3. Autophagy 3: 452-460, 2007.

18. Werner K, Lademann F, Thepkaysone ML, Jahnke B, Aust DE, Kahlert C, Weber G, Weitz J, Grützmann R and Pilarsky C: Simultaneous gene silencing of KRAS and anti-apoptotic genes as a multitarget therapy. Oncotarget 26: 3984-3992, 2016.

19. Rückert F, Samm N, Lehner AK, Saeger HD, Grützmann R and Pilarsky C: Simultaneous gene silencing of Bcl-2, XIAP and Survivin re-sensitizes pancreatic cancer cells towards apoptosis. BMC Cancer 10: 379, 2010.

20. Welsch T, Younsi A, Disanza A, Rodriguez JA, Cuervo AM, Scita G and Schmidt J: Eps8 is recruited to lysosomes and subjected to chaperone-mediated autophagy in cancer cells. Exp Cell Res 316: 1914-1924, 2010.

21. Furuta S, Hidaka E, Ogata A, Yokota S and Kamata T: Ras is involved in the negative control of autophagy through the class I PI3-kinase. Oncogene 23: 3898-3904, 2004

22. Kim SE, Park HJ, Jeong HK, Kim MJ, Kim M, Bae ON and Baek SH: Autophagy sustains the survival of human pancreatic cancer PANC-1 cells under extreme nutrient deprivation conditions. Biochem Biophys Res Commun 463: 205-210, 2015.

23. Haq R and Fisher DE: Biology and clinical relevance of the micropthalmia family of transcription factors in human cancer. J Clin Oncol 29: 3474-3482, 2011.

24. Perera RM, Stoykova S, Nicolay BN, Ross KN, Fitamant J, Boukhali M, Lengrand J, Deshpande V, Selig MK, Ferrone CR, et al: Transcriptional control of autophagy-lysosome function drives pancreatic cancer metabolism. Nature 524: 361-365, 2015.

25. Son J, Lyssiotis CA, Ying H, Wang X, Hua S, Ligorio $M$, Perera RM, Ferrone CR, Mullarky E, Shyh-Chang N, et al: Glutamine supports pancreatic cancer growth through a KRASregulated metabolic pathway. Nature 496: 101-105, 2013.

26. Ying H, Kimmelman AC, Lyssiotis CA, Hua S, Chu GC, FletcherSananikone E, Locasale JW, Son J, Zhang H, Coloff JL, et al: Oncogenic Kras maintains pancreatic tumors through regulation of anabolic glucose metabolism. Cell 149: 656-670, 2012. 
27. Bryant KL, Mancias JD, Kimmelman AC and Der CJ: KRAS: Feeding pancreatic cancer proliferation. Trends Biochem Sci 39: 91-100, 2014.

28. Commisso C, Davidson SM, Soydaner-Azeloglu RG, Parker SJ, Kamphorst JJ, Hackett S, Grabocka E, Nofal M, Drebin JA, Thompson CB, et al: Macropinocytosis of protein is an amino acid supply route in Ras-transformed cells. Nature 497: 633-637, 2013.

29. Guo JY, Chen HY, Mathew R, Fan J, Strohecker AM, KarsliUzunbas G, Kamphorst JJ, Chen G, Lemons JM, Karantza V, et al: Activated Ras requires autophagy to maintain oxidative metabolism and tumorigenesis. Genes Dev 25: 460-470, 2011.
30. Guo JY, Xia B and White E: Autophagy-mediated tumor promotion. Cell 155: 1216-1219, 2013.

31. Moore PS, Sipos B, Orlandini S, Sorio C, Real FX, Lemoine NR, Gress T, Bassi C, Klöppel G, Kalthoff H, et al: Genetic profile of 22 pancreatic carcinoma cell lines. Analysis of K-ras, p53, p16 and DPC4/Smad4. Virchows Arch 439: 798-802, 2001. 